\title{
Avaliação de dinapenia e associação com função tireoidiana em idosos da Universidade Aberta à Terceira Idade - UATI
}

\author{
Evaluation of dynapenia and association with thyroid function in elderly at the Open \\ University of the Elderly - UATI
}

Jocyel de Brito Oliveira ${ }^{1 *}$, Helton Estrela Ramos ${ }^{2}$

${ }^{1}$ Mestrando em Processos Interativos de Órgãos e Sistemas, Universidade Federal da Bahia.

${ }^{2}$ Professor Associado do Departamento de Biorregulação e do Programa de Pós-Graduação Processos Interativos dos Órgãos e Sistemas UFBA.

\begin{abstract}
Resumo
Introdução: o presente artigo analisou e avaliou a prevalência de dinapenia em associação da idade dos Hormônio Estimulador da tireoide (TSH) e $\mathrm{T}_{3}$ em idosos da Universidade Aberta à Terceira Idade - UATI. A tireoide produz e secreta os hormônios triiodotironina $\left(\mathrm{T}_{3}\right)$ e tiroxina $\left(\mathrm{T}_{4}\right)$, responsáveis por controlar o metabolismo celular. $\mathrm{O}$ termo dinapenia tem sido utilizado para descrever a diminuição da força muscular relacionada à idade separando desta forma, a dinapenia da redução da massa muscular. Metodologia: trata-se de um estudo de corte transversal que investigou 63 mulheres com idade entre 60 e 95 anos, resultando numa idade média das participantes foi de 69,6 anos, não institucionalizadas, matriculados no projeto "Universidade Aberta à Terceira Idade (UATI)", da Universidade Estadual da Bahia (UNEB). A força de preensão palmar (FPP) foi avaliada em quilograma ( $\mathrm{kg}$ ), por meio do dinamômetro digital manual da marca INSTRUTHERM. Resultados: foram diagnosticados com dinapenia as pacientes que possuíram a FPP inferior a $20 \mathrm{~kg}$. Descobriu-se que 23 idosas (36,51\%) foram diagnosticadas com dinapenia. A correlação entre as variáveis TSH e dinapenia foi positiva e fraca Spearman=0.17 ( $p$-valor= 0.22). Assim, a relação entre o FPP e a idade não parece ser linear possuindo uma correlação negativa e fraca: Spearman= -0.11 ( $p$-valor= 0.39). Conclusão: o nível sérico de TSH e idade não tiveram associação significativa com a presença de dinapenia. Houve associação entre $T_{3}$ sérico e dinapenia, porém não é possível identificar em qual quartil está essa associação.

Palavras-chave: Dinapenia. Idoso. TSH. T Tireoide.
\end{abstract}

\begin{abstract}
Introduction: this article analyzed and evaluated the prevalence of dynapenia in association with the age of thyroid stimulating hormones (TSH) and T3 in the elderly at Universidade Aberta do Idoso-UATI. The thyroid produces and secretes the hormones triiodothyronine $\left(T_{3}\right)$ and thyroxine $\left(T_{4}\right)$ responsible for the control of cellular metabolism. The term dynapenia has been used to describe the decrease in muscle strength related to age, separating dynapenia from the reduction of muscle mass. Methodology: this is a cross-sectional study that investigated 63 women between 60 and 95 years old, resulting in an average age of participants of 69.6 years old, non-institutionalized, registered in the project the Open University Of The Elderly (UATI)", of Bahia State University (UNEB). The Hand Grip Strength (HGS) was measured in kilograms (kg), using the INSTRUTHERM manual digital dynamometer. Results: patients with FPP less than $20 \mathrm{~kg}$ were diagnosed with dynapenia. It was found that 23 elderly women (36.51\%) were diagnosed with dynapenia. The correlation between TSH and dynapenia variables was positive and weak Spearman $=0.17(p$-value $=0.22)$. Thus, the relationship between (FPP) and age does not appear to be linear with a weak and negative correlation: Spearman $=-0.11$ ( $p$-value $=0.39)$. Conclusion: the serum TSH level and age had no significant association with the presence of dynapenia. There was an association between $\left(T_{3}\right)$ and serum dinapenia, but it is not possible to identify in which quartile this association is found. Keywords: Dynapeni. Old man. TSH. T. $_{3}$. Thyroid.
\end{abstract}

\section{INTRODUÇÃO}

O Brasil está em processo de transição demográfica, com alterações no padrão de morbimortalidade e aumento na prevalência de doenças crônico-degenerativas e incapacitantes (VERAS, 2009). Dessa forma, as mudanças demográficas gradualmente tornam-se mais relevantes para compreender os fatores de risco modificáveis para deficiência e perda de independência com o avançar da idade (MITCHELL et al., 2012).

Correspondente/Corresponding: *Jocyel de Brito Oliveira - End: Av. Paulino de Andrade, n 212 Santo Amaro, BA - Tel: 71 99933-3235 - E-mail: jocyeldebritooliveira@hotmail.com
Nesse sentido, os problemas de saúde dos idosos desafiam os modelos de cuidado na medida em que a sociedade envelhece (VERAS, 2012). Sabendo que o envelhecimento é um processo inevitável e irreversível, no qual o indivíduo torna-se mais frágil aparecendo declínios psicológicos, sociais, fisiológicos, funcionais e várias limitações (ROSA, 2012).

Força muscular é utilizado para designar a habilidade de um determinado músculo, ou grupamento muscular, em produzir ou resistir a uma força; podendo ser classificada como isométrica, isocinética ou isotônica (SCHLÜSSEL, 2008). Pode-se afirmar que o termo dinapenia tem sido utilizado para descrever a diminuição da força muscular 
relacionada à idade, diferenciando, desta forma, a dinapenia da redução da massa muscular (MANINI; CLARK, 2011). A dinapenia decorre de uma combinação da evolução da sarcopenia e de alterações do sistema nervoso central. É a primeira e a mais importante manifestação clínica da síndrome da fragilidade do idoso (SFI) (IWAMURA, 2017), podendo servir de preditor de incapacidade e mortalidade (NEWMAN, 2006).

A tireoide, através da produção e secreção dos hormônios triiodotironina $\left(T_{3}\right)$ e tiroxina $\left(T_{4}\right)$, desempenham significativo papel no controle do metabolismo corporal (BUESCU; GREGO FILHO, 2001). A produção dos hormônios tireoidianos (HTs) é controlada pelo TSH sintetizado pela parte anterior da glândula hipófise em resposta ao hormônio liberador da tireotrofina (TRH), secretado pelo hipotálamo (RAUEN, 2011). O músculo esquelético é alvo relevante da ação do hormônio tireoidiano (HT). Os HTs exercem influências importantes na bioquímica das células musculares, incluindo a composição da cadeia pesada da miosina, o metabolismo das proteínas, as proteínas reguladoras da miofibrina, o cálcio e o metabolismo energético (CAIOZZO; BAKER; BALDWIN, 1998).

Tratando-se de envelhecimento e todas as questões metabólicas envolvidas é necessário entender que todo organismo sofrerá possíveis alterações hormonais. A possibilidade de disfunção aumenta com o envelhecimento, o que também se verifica pelo aumento da prevalente condição denominada de hipotireoidismo subclínico (HSC) (SURKS, 2004). Assim, várias mudanças nas concentrações dos HTs ocorrem durante o envelhecimento como a queda na concentração dos níveis séricos de $\mathrm{T}_{3}$ em idosos (MARIOTTI et al., 1995).

O presente artigo analisou a prevalência de dinapenia e avaliação da sua associação com idade, $\mathrm{TSH}_{\mathrm{H}} \mathrm{T}_{3}$ séricos em idosos matriculados no programa Universidade Aberta à Terceira Idade - UATI, da Universidade Estadual da Bahia (UNEB), Salvador, Bahia.

\section{METODOLOGIA}

Trata-se de estudo de corte transversal que investigou 63 mulheres com idade entre 60 e 95 anos, não institucionalizadas, matriculadas no projeto UATI, da UNEB, que tenham assinado o termo de consentimento livre esclarecimento (TCLE).

Foram excluídos da pesquisa os idosos que possuíam doença mental, diabetes mellitus descompensado (Glicemia de jejum > $180 \mathrm{mg} / \mathrm{Dl}$ ou hemoglobina glicada/A1C > 8.5\%), Hipertensão Arterial Sistêmica (HAS) [moderada ou grave - Pressão Arterial (PA) sistólica maior que $160 \mathrm{mmHg}$ e/ou PA diastólica maior que $90 \mathrm{mmHg}$ ], doença aguda grave, doença hepática ou renal crônica, histórico de doença maligna, uso de medicamentos que alteram a função tireoidiana (levotiroxina, amiodarona, lítio, carbamazepina, AAS, bromoergocriptina, propranolol, heparina, corticoide, quimioterápico, interferon-alfa, interleucina-2, reposição hormonal com estrogênio, tratamento para câncer de próstata), diagnóstico de hipotireoidismo, diagnóstico de hipertireoidismo, presença de limitações físicas e psicoló- gicas que impeçam a execução dos protocolos adotados no estudo, distúrbios neurológicos graves, histórico de acidente vascular cerebral (AVC), incapacidade de coleta de sangue venoso, idosos que não consigam/possam ficar em pé e tenha realizado exame com contraste iodado nos últimos dois meses (tomografia, etc.).

Toda dosagem hormonal foi realizada no DNA Laboratório e genética médica; foram coletados dois tubos de sangue total para dosagens laboratoriais e função tireoidiana completa: Hemograma, Glicemia, Hemoglobina glicada, Creatinina, Testes de Função Tireoidana (Hormônio estimulador da Tireoide, TSH; Tiroxina livre, T4L; Triiodotirinina Livre, T3L; T3 reverso; ATPO, anticorpo anti-peroxidase; AATG, anticorpo anti-tireoglobulina) e Vitamina D. Os valores de referências usados para o TSH foi $(0,27-4,2 \mathrm{mU} / \mathrm{L})$, para o $\mathrm{T}_{3}(2,0-4,4 \mathrm{pg} / \mathrm{mL})$ e $\mathrm{T}_{4}(0,93-1,7 \mathrm{ng} / \mathrm{dL})$.

A Força de Preensão Palmar (FPP) foi avaliada em quilograma (kg), por meio do dinamômetro digital manual da marca INSTRUTHERM. Foram diagnosticados com dinapenia as pacientes que possuíram a FPP inferior a $20 \mathrm{~kg}$ para mulheres, o que expressa indicador de pior mobilidade comparado a diminuição da massa muscular (LAURETANI et al., 2003). Este valor foi o maior entre as duas tentativas (ALEXANDRE, 2014).

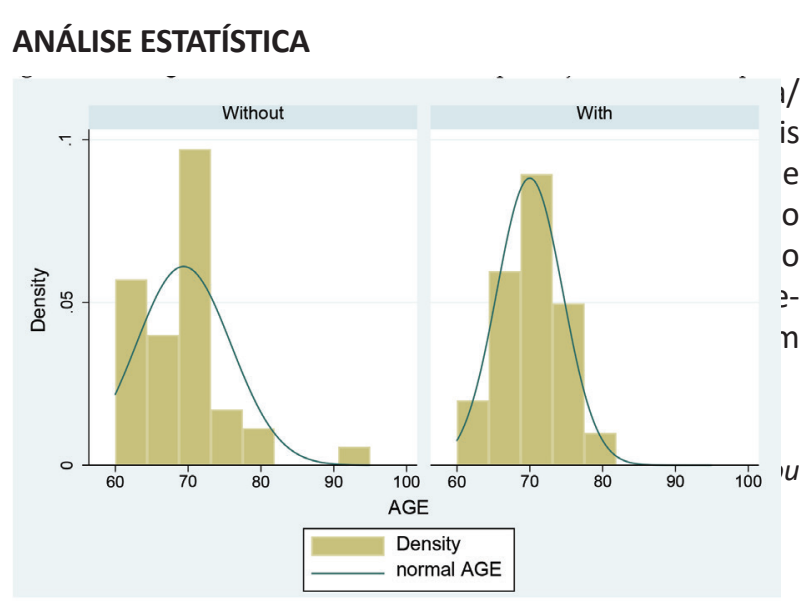

Fonte: Projeto Sarcopenia

Em seguida, avaliou-se a associação entre os quartis de TSH, de $\mathrm{T}_{3}$ e o desfecho através do Teste Exato de Fisher, o qual, foi utilizado como alternativa ao Teste de Associação Qui-Quadrado por conta do não cumprimento de pré-requisitos necessários. As análises foram processadas no software estatístico STATA versão 10 e o nível de significância adotado foi de $5 \%$.

\section{RESULTADOS}

A idade média das participantes foi de 69,6 anos. Das 63 idosas pesquisadas, 23 (36,51\%) foram diagnosticadas com dinapenia. Para verificar a diferença na idade para aquelas idosas que possuem ou não dinapenia, o teste Mann-Whitney foi performado e o P-valor $=0.50$ sugere que essa distinção não é estatisticamente significante, ou 
seja, as idosas com e sem dinapenia não possuem diferença de idade.

A análise dos quartis de TSH associados à dinapenia mostrou que não existe evidência de significância estatística, no qual, o P-valor corresponde a 1.000 (Figura 2).

Figura 2 - Associação dinapenia e quartis de TSH

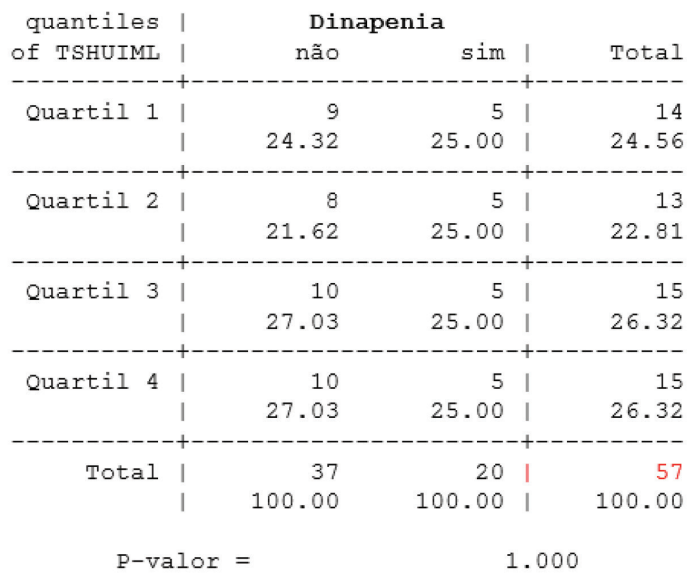

Fonte: Projeto Sarcopenia

Observando os resultados da associação entre a variável quartis de $\mathrm{T}_{3}$ sérico e o desfecho, observa-se um p-valor $=0.030$, indicando a existência de associação entre os quartis de $T_{3}$ sérico e dinapenia, mas não se pode inferir qual quartil está ou não associado com a presença de dinapenia (Figura 3).

Figura 3 - Associação dinapenia e quartis de T3

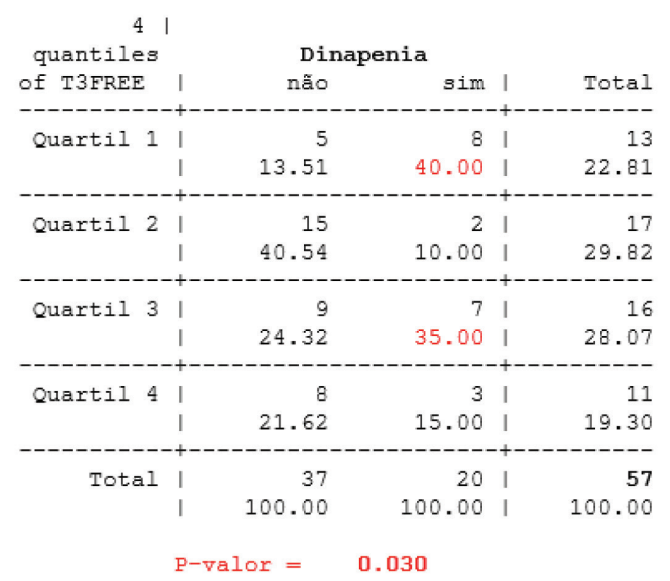

Fonte: Projeto Sarcopenia

O gráfico de dispersão na relação TSH - idade, apresenta uma relação que parece ser um pouco linear correlação positiva e fraca, conforme expressa a figura 4 . O p-valor do Spearman diz que se a correlação for ser baixa, não é estatisticamente diferente de zero e por isso não é considerável.
Figura 4-Gráfico de dispersão para avaliar a relação TSHX Idade

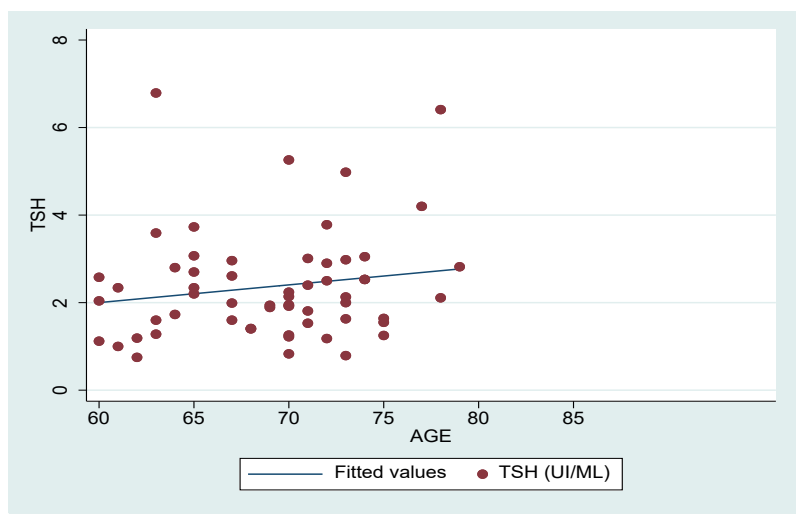

Fonte: Projeto Sarcopenia

O gráfico de dispersão entre o FPP e a idade indica uma relação não linear com correlação negativa e fraca, no qual, os resultados de Spearman= -0.11 ( $p$-valor= 0.39). (Figura 5)

Figura 5- Gráfico de dispersão para avaliar a relação FPP (kg) $x$ Idade

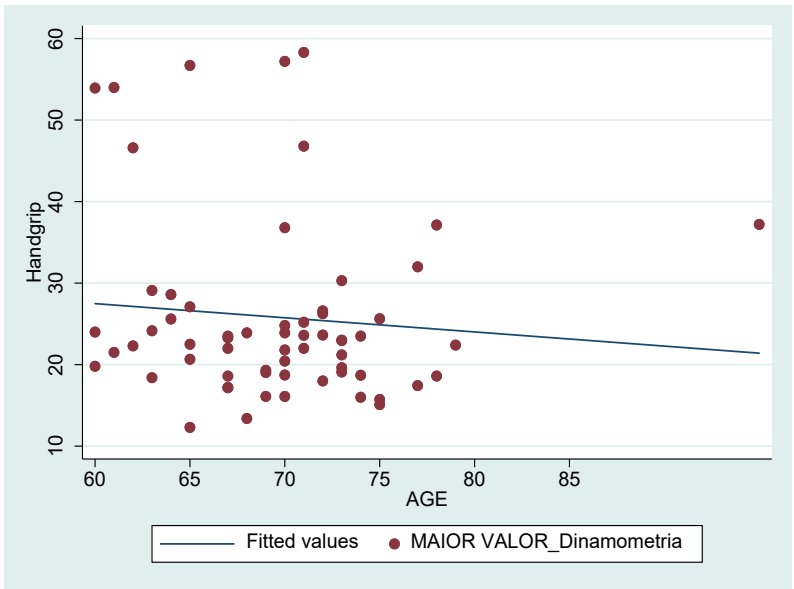

Fonte: Projeto Sarcopenia

\section{DISCUSSÃO}

Este estudo mostra que $36,51 \%$ das idosas possuem dinapenia, com FPP média de $24,44 \mathrm{~kg}$. A redução da força com o avanço da idade é um fenômeno que pode levar ao declínio na execução das atividades diárias normais (BARBOSA et al., 2006). A redução de massa muscular (sarcopenia) pode gerar limitações funcionais (dinapenia) que acarretam perda da independência, quedas e fraturas (ROSENBERG, 1997).

Não houve associação significativa entre TSH e a presença de Dinapenia. Várias mudanças ocorrem no hormônio da tireoide durante o envelhecimento: diminuição nas concentrações séricas de TSH em idosos saudáveis, os níveis de $\mathrm{T}_{3}$ livre demonstram um declínio claro depen- 
dente da idade, enquanto as concentrações séricas total e livre do $\mathrm{T}_{4}$ (FT4) permanecem inalterados (MARIOTTI, 1995). O TSH sérico também pode diminuir com a idade, doença ou drogas (VAN DEN BELD et al, 2005). Houve associação entre os quartis de $T_{3}$ e a variável dinapenia. Entretanto, não foi possível inferir em qual quartil está ou não associado com a presença de dinapenia.

O p-valor do Spearman diz que a correlação, por ser baixa, não é estatisticamente diferente de zero e por isso não é importante. Quanto menor o p-valor mais indicativo tem de que a correlação entre as variáveis é significativa.

Força de preensão menor está associada ao aumento da mortalidade por todas as causas, a baixa massa muscular (avaliada por tomografia computadorizada e densitometria óssea DXA), não estava fortemente relacionada à mortalidade (NEWMAN, 2006).

\section{CONCLUSÃO}

Constatou-se que em idosas matriculadas na UATI (UNEB) não houve associação entre níveis de TSH sérico e presença de dinapenia. Tendo em vista os aspectos estatísticos observados é possível indicar que não houve valor significante entre a relação FPP versus idade, por ter apresentado uma correção negativa e fraca.

Existe associação entre os quartis de $T_{3}$ com a variável dinapenia, entretanto não é possível identificá-las, tornando-se necessário aumentar o número das amostras para ampliar os resultados.

\section{REFERÊNCIAS}

ALEXANDRE, T. S. et al. Sarcopenia according to the european working group on sarcopenia in older people (EWGSOP) versus Dynapenia as a risk factor for disability in the elderly. J. Nutr. Health Aging, New York, v.18, v.5, p. 547-553, 2014.

BARBOSA, A. R. et al. Relação entre estado nutricional e força de preensão manual em idosos do município de São Paulo, Brasil: Dados da pesquisa SABE. Revista Brasileira de Cineantropometria e Desempenho Humano, Santa Catariana, v.8, n.01, p 38-43, 2006.

BUESCU, A.; GREGO-FILHO, J. Tireóide. Propedêutica clínica, laboratorial e imaginológica. In: CORONHO, V. et al. (Eds.) Tratado de endocrinologia e cirurgia endócrina. Rio de Janeiro: Guanabara Koogan, 2001.p. 359368.

CAIOZZO, V. J.; BAKER, M. J.; BALDWIN, K. M. Novel transitions in MHC isoforms: separate and combined effects of thyroid hormone and mechanical unloading. J. Appl. Physiol., Washington, v. 85, p. 22372248,1998 .

CLARK, B.C.; MANINI, T.M. Sarcopenia Dynapenia. J. Gerontol. A Biol. Sci. Med. Sci., Washington, v.63, n.8, p. 829-34, 2008.

IWAMURA, M.; KANAUCHI, M. A cross-sectional study of the association between dynapenia and higher-level functional capacity in daily living in communitydwelling older adults in Japan. BMC Geriatr.. London, v.17, n.1, p.1-6, 2017.

LAURETANI, F. et al. Age-associated changes in skeletal muscles and their effect on mobility: an operational diagnosis of sarcopenia. J. Appl. Physiol., Washington, v.95, p. 1851-1860, 2003.

MARIOTTI, S. et al. The aging thyroid. Endocr. Rev., Baltimore, v.16, n. 6, p. 686-715,1995.

MITCHELL, W. K. et al. Sarcopenia, dynapenia, and the impact of advancing age on human skeletal muscle size and strength; a quantitative review. Front. Physiol., Basel, v.11, n.3, n.1-18, 2012.

NEWMAN, A.B. et al. Strength, but not muscle mass, is associated with mortality in the health, aging and body composition study cohort. J. Gerontol, A Biol, Sci, Med, Sci., , Washington v.61, n.1, p.72-77, 2006.

RAUEN, G. et al. Abordagem do hipotireoidismo subclínico no idoso. Rev. Bras. Clin. Med., São Paulo, v.9, n.4, p.294-299, jul./ago. 2011.

ROSA, B. P. de S. et al. Envelhecimento, Força Muscular E Atividade Física: Uma Breve Revisão Bibliográfica. Revista Científica FacMais, [s.l], v. 2, p. 141-152, 2012.

ROSENBERG, I. H. Sarcopenia: origins and clinical relevance. J. Nutr., Philadelphia, v.127, n.5, p.990S-1S, 1997.

SCHLÜSSEL, M. M. A dinamômetria manual e seu uso na avaliação nutricional. Revista de Nutrição, Campinas, v. 21, n. 2, p. 223-235, abr./mar. 2008.

SURKS, M. I. et al. Subclinical thyroid disease: scientific review and guidelines for diagnosis and management. JAMA, Rio de Janeiro, v. 291, n.2, p.228-240, 2004.

VAN DEN BELD, A. W. et al. Thyroid hormone concentrations, disease, physical function, and mortality in elderly men. J. Clin. Endocrinol. Metabol., [s.I], v.90, n.12, p.6403-6409, 2005.

VERAS, R. O envelhecimento populacional contemporâneo: demandas, desafios e inovações. Rev. Saúde Pública, São Paulo, v. 43, n.3, p.548554, 2009.

VERAS, R. P. et al. Prevenção de doenças em idosos: os equívocos dos atuais modelos. Cad. Saúde Pública, Rio de Janeiro, v. 28 , ed. 10, p. 1834-1840, 2012.

Submetido em: 07/12/2020

Aceito em: 14/12/2020 